\title{
La asertividad de China en el ámbito de la Seguridad internacional y su posible impacto en Sudamérica
}

China's assertiveness in the field of International Security and its possible impact on South America

Emilse E. Calderón ${ }^{1}$

\section{Resumen}

El análisis de las tendencias geopolíticas mundiales, suelen brindarle poca atención al impacto sobre América Latina, en alguna medida, dado el supuesto de la irrelevancia estratégica o a la condición de patio trasero norteamericano que tiene la región. No obstante, en función de la inserción internacional que ha desplegado China durante los últimos años y el acercamiento a estas latitudes, deviene necesario posicionar el foco de reflexión en las consecuencias que tal proximidad puede conllevar para los estados de la subregión. Más específicamente y partiendo de la importancia de comprender los procesos en curso que se despliegan en el contexto sudamericano, el presente escrito examina las implicancias que posee la mayor asertividad de China en el ámbito de la seguridad internacional, expresada en sus capacidades estratégicomilitares y en su voluntad de participar en la gobernanza de la agenda global, sobre los estados de América del Sur. Esto en función de sustentar la premisa sobre la situación de extrema vulnerabilidad que atraviesa la subregión frente a una posible escalada de tensión en el vínculo entre Washington y Beijing. Finalmente, el artículo deja traslucir una mirada teórico-conceptual interméstica y se desarrolla a través del empleo de una metodología cualitativa de investigación.

Palabras clave: China, Sudamérica, Seguridad internacional, Estados Unidos, Geopolítica

\section{Abstract}

Analysis of global geopolitical trends tends to pay little attention to the impact on Latin America, to some extent, given the assumption of strategic irrelevance or the

Recibido: 8 de julio de 2021 Aceptado: 20 de diciembre de 2021 Publicado: 7 de enero de 2022

${ }^{1}$ Doctora en Relaciones Internacionales, Investigadora del Consejo Nacional de Investigaciones Científicas y Técnicas (CONICET) de Argentina y profesora de la Facultad de Ciencia Política y Relaciones Internacionales de la Universidad Nacional de Rosario (UNR). Correo electrónico: emilse.calderon@fcpolit.unr.edu.ar ID https://orcid.org/0000-0002-2975-0572 
region's condition of the North American backyard. However, depending on the international insertion that China has deployed in recent years and the approach to these latitudes, it becomes necessary to position the focus of reflection on the consequences that such proximity may entail for the states of the subregion. More specifically, and based on the importance of understanding the ongoing processes that are unfolding in the South American context, this paper examines the implications of China's greater assertiveness in the field of international security, expressed in its strategic-military and military capabilities. In their willingness to participate in the governance of the global agenda, on the states of South America. This is based on supporting the premise of the extremely vulnerable situation that the subregion is experiencing in the face of a possible escalation of tension in the link between Washington and Beijing. Finally, the article reveals an intermestic theoretical-conceptual look and is developed through the use of a qualitative research methodology.

Keywords: China, South America, International Security, United States, Geopolitics

\section{Introducción}

En los albores de la tercera década del siglo XXI las tendencias geopolíticas mundiales parecen cobrar nitidez al tiempo que permiten avizorar momentos de mayor incertidumbre. Luego de un largo período de posguerra fría en el que se sucedieron cambios con relativa celeridad en la estructura de poder vinculada a distintos ámbitos de las Relaciones Internacionales, se perfilan con bastante precisión los estados de mayor peso y sus políticas en el ámbito estratégico-militar. Asimismo, ese perfilamiento implica la contraposición de los intereses vitales de las potencias en diversos escenarios en todo el globo con el consecuente anuncio de tensiones, rispideces y hasta la posibilidad de conflictos.

A grandes rasgos se pueden identificar cuatro tendencias. La primera es la mayor asertividad de China en el ámbito de la Seguridad internacional expresada en sus capacidades estratégico-militares y en su voluntad de participar en la gobernanza de la agenda global relativa a esos asuntos, lo cual permite prever una coexistencia compleja con Estados Unidos. La segunda tendencia es el retroceso relativo de Washington frente al avance de las capacidades militares y tecnológicas de China y Rusia junto con la erosión de su liderazgo en la gobernanza de dicha agenda global. En tercer lugar se encuentra la re-emergencia de Rusia como polo de poder mundial independientemente de los cuestionamientos que existen al respecto de la solidez de 
su desarrollo económico y social y de su imagen internacional. Finalmente, una cuarta tendencia está marcada por el doble juego de la Unión Europea entre las intenciones de Estados Unidos de reapuntalar la Alianza del Atlántico Norte (OTAN) recuperando su compromiso con los valores democráticos en contraposición a China, y sus estrechos lazos con Beijing dada su necesidad de acomodarse en un contexto de transición en el orden internacional y entendiendo que el máximo desafío para su seguridad es Moscú.

No obstante, cuando se reflexiona acerca de dichas tendencias geopolíticas en el contexto de la reconfiguración del sistema internacional y el orden en términos estratégico-militares, no siempre se analiza el impacto sobre América Latina o esta tarea se lleva a cabo de manera relativamente escueta, con un final que indefectiblemente gira en torno a la irrelevancia estratégica o a la condición de patio trasero norteamericano que tiene la región. En función de ello y partiendo de la importancia de comprender el estado de situación que se desarrolla en la subregión, el presente escrito tiene como propósito examinar las implicancias para Sudamérica de la primera de las tendencias geopolíticas explicitadas, considerando en términos generales e implícitamente al resto, como partes de un contexto sistémico único. En línea con este objetivo la premisa a sustentar indica que, dado los procesos intra subregionales en curso, la situación de América del Sur es de extrema vulnerabilidad frente a una posible escalada de tensión en el vínculo entre Estados Unidos y China en el ámbito de la Seguridad internacional porque sus posibilidades de mantenerse al margen de la puja de poder entre ambas potencias son mínimas.

En consonancia con este planteo, prepondera la mirada teórico-conceptual interméstica (Rose 1998) dada su mayor pertinencia para recuperar los procesos por los que transitan los estados sudamericanos y determinan la situación que atraviesa la subregión en su conjunto, sin ignorar el impacto de las variables sistémicas. Asimismo, en función de la especificidad que presenta la problemática de seguridad en los países periféricos del sistema internacional, se rescata el espíritu de los aportes de autores como M. Ayoob (1995), S.Neumann (1995), O. Holsti(1995), C. Escudé (1995), A. Acharya (1999), entre otros, que afirman la preponderancia del condicionamiento de la variable doméstica por sobre la sistémica.

Por último, cabe especificar que se confeccionan tres apartados. El primero relativo a la explicitación de los rasgos de la tendencia geopolítica a examinar, otro abocado a la revisión de los procesos en curso a nivel subregional y una tercera sección dedicada a sustentar la premisa propuesta. Finalmente, se contará con un apartado conclusivo direccionado a retomar las ideas más importantes del escrito. 


\section{China y la Seguridad internacional: un ascenso planificado en el contexto de un descenso no querido}

La puja de poder entre un Estados Unidos en retroceso relativo y una China ya emergida y posicionada como una potencia global, es el rasgo principal que presenta el nivel sistémico de las Relaciones Internacionales de la segunda década del siglo XXI, independientemente de las disquisiciones con respecto a las características e implicancias de dicha puja para el devenir del sistema y del orden internacional. La misma, además, se despliega preponderantemente en el ámbito político-diplomático, tecnológico y económico-comercial. No obstante, se puede observar como tendencia geopolítica la mayor asertividad de Beijing en el ámbito de la seguridad internacional expresado en sus capacidades estratégico-militares y en su voluntad de participar en la gobernanza de la agenda global de tales cuestiones, lo cual permite avizorar una coexistencia compleja y tensa con Washington.

En este sentido China, al mismo tiempo que despliega su teoría de desarrollo pacífico en las relaciones con los estados de Asía y el Pacífico, desde los albores del siglo XXI ha ampliado sus capacidades de poder duro. Específicamente aumentó aquellas ligadas al ámbito naval e inició un proceso de proyección de poder en lo que denomina su línea marítima vital para garantizar su presencia en el Mar de China y el paso por el Estrecho de Malaca ${ }^{2}$. Esto se encuentra en sintonía con su percepción de Estados Unidos como potencia equivalente a la cual debe negarle las posibilidades de alcanzar la supremacía regional y con el desplazamiento a un lugar secundario de susesfuerzos por establecer un orden sistémico en Asia, contemplativo con respecto a las preocupaciones e intereses del resto de los países de la región (Panda, 2018). De hecho el Libro Blanco sobre Defensa Nacional de China de 2019 reconoce la emergencia de una nueva era caracterizada por el multipolarismo y que tiene a China como uno de los 4 actores más importante, además de Estados Unidos, Rusia y la Unión Europea y que se caracteriza por el incremento de la competición a nivel político-estratégico (Depto. Seguridad Nacional, 2019).

En lo que respecta a las capacidades de poder duro, Beijing aplica la estrategia Near Seas Active-Defense Capabilities que, a contramano del desarrollo de una marina de guerra defensiva como se planteó desde los años 80 , se direcciona a incorporar sistemas de armas aptos para implementar una estrategia Anti-Access Area-Denial mediante un proceso de modernización (Baqués Quesada, 2019). Esta estrategia tiene por objetivo obligar a los rivales potenciales de China que se acerquen

\footnotetext{
${ }^{2}$ El $80 \%$ de los hidrocarburos que importa China llegan por mar a través del estrecho de Malaca, al tiempo que es la vía por la cual exporta la mayoría de sus manufacturas.
} 
a sus costas a soportar pérdidas materiales y humanas importantes en poco tiempo y el desarrollo de capacidades que le es inherente se explicita en el Libro Blanco sobre Defensa Nacional de China de $2015^{3}$. De este modo, Beijing da cuenta de su vocación defensiva, estando dispuesta a responder militarmente solo en caso de ser atacada, y su preocupación por mantener la estabilidad en las regiones fronterizas y cercanas a su influencia creando un ambiente pacífico como condición para el desarrollo económico.

No obstante y si bien dicho documento no establece metas expansivas, es imposible desconocer el proceso de proyección de poder que China ha ido desplegando en su línea marítima vital en respuesta a su objetivo tradicional de lograr la autosuficiencia y asegurar el abastecimiento de recursos mediante la seguridad de las rutas marítimas. Incluso, Beijing inicia este camino mucho antes, con la reforma de la Ley del Mar Territorial de 1992 que le permitió ampliar sus reclamos territoriales de manera meridianamente sólida, instrumentando medidas de presión moderadas para no generar un enfrentamiento abierto con Estados Unidos o Japón, pero contundentes (Baqués Quesada, 2019). Por otra parte, China desarrolla una estrategia de consolidación de facilidades portuarias en distintos estados de Asía y África que, a excepción de Djibouti, son en su origen bases de apoyo logístico y dejan traslucir una cadena de alianzas con varios países. Finalmente se ha ido estableciendo, también, a lo largo de la costa asiática desde el Mar Rojo y el Golfo Pérsico hasta el Mar de China Meridional y Oriental, una serie de enclaves estratégicos (bases navales y aéreas, puertos o prospecciones petrolíferas) en territorios propios y de países amigos, formando el conocido Collar de Perlas que apunta al control del Océano Índico. Este deviene fundamental para acompañar la iniciativa One Belt One Road (OBOR) en lo que respecta a la concreción del proyecto marítimo denominado Ruta de la Seda Marítima, complementario de su contraparte terrestre conocido como Cinturón Económico de la Ruta de Seda (Girado, 2017).

Por otra parte, en lo que se refiere a la gobernanza de la agenda global de Seguridad internacional, China ha dado muestras incipientes pero certeras de su vocación por ampliar su participación en congruencia con el alcance global de sus intereses y su disposición a defenderlos. Los cursos de acción más representativos en este sentido se articulan en primer lugar en torno a su rol en el marco de las

\footnotetext{
${ }^{3}$ China proyecta una flota de alta mar para acceder a las cadenas de islas, desde las más cercanas a sus costas hasta las más lejanas y, en sintonía con ello, se inicia el programa de construcción de portaaviones. Asimismo, los diseños nuevos de las fuerzas anfibias con una capacidad de asalto significativa, el crecimiento exponencial de las fuerzas de infantería de marina, los buques de superficie con capacidad de escolta y combate y los sistemas de detección modernos, artillería de largo alcance, misiles de crucero y helicópteros embarcados y la multiplicación del potencial de la fuerza submarina (Baqués Quesada, 2019).
} 
Operaciones para el Mantenimiento de la Paz (OMP) de Naciones Unidas (NU). A través de las OMP como ventana al mundo para mostrar el compromiso de los estados con la paz y seguridad internacional, Beijing busca proyectar una imagen de potencia global responsable y reducir la percepción que se tiene del país como una amenaza al orden democrático liberal occidental. De este modo, China ha incrementado progresiva y constantemente su compromiso con NU desde 2003 y con la llegada de Xi Jinping. Puntualmente, a partir de 2015 se da un salto cualitativo anunciando la creación de una fuerza permanente para el Mantenimiento de la Paz compuesta de 8000 soldados y el despliegue de su primer batallón de infantería para proteger a los ingenieros y personal médico en Sudán del Sur (Hevia Fernández, 2020). A ello se adiciona la donación de 100 millones de dólares a la Unión Africana en los siguientes 5 años para apoyar la construcción de una Fuerza Africana de Reserva y la Capacidad Africana para la Respuesta Inmediata a las Crisis y la creación en 2016 del Fondo Fiduciario de las NU para la Paz y el Desarrollo, comprometiéndose con la contribución de 200 millones de dólares en un período de 10 años(Hevia Fernández, 2020).En consonancia con esto, para 2021 Beijing se transformó en el segundo mayor contribuyente financiero de las OMP luego de Estados Unidos aportando un 15,21\% del presupuesto (UN, 2021a) y es de esperar que tenga mayor influencia sobre las futuras operaciones y la forma en que se llevarán a cabo. Asimismo, participa en 8 de las 14 operaciones en curso y en 5 de ellas despliega tropas alcanzando un total de 2544 efectivos siendo el miembro permanente del Consejo de Seguridad que más militares aporta (UN, 2021b).

En segunda instancia se encuentra el apoyo dado a la noción de Protección Responsable en el marco de la cuestionada aplicación de la doctrina de Responsabilidad de Proteger (RtoP) en Libia ${ }^{4}$. Esto se produce a través de su incorporación en algunos discursos de funcionarios explicitando la voluntad de China de poner en valor sus preferencias normativas luego de su infructuosa relación con la RtoP. Cabe recordar que luego de la fuerte resistencia entre 2001 y 2005, Beijing aprobó dicha norma pero enfatizando los pilares relativos a la responsabilidad de los estados y de la comunidad internacional y minimizando el potencial del tercer pilar referido al deber de la comunidad internacional de adoptar las acciones colectivas apropiadas frente a la afectación de la seguridad de cierta población. Finalmente, con posterioridad al desenlace del escenario en Libia, en el marco del cual Beijing apoyó

\footnotetext{
${ }^{4}$ En 2012 apareció el concepto de Protección Responsable en un artículo periodístico de Ruan Zongze que es el vicepresidente de un think-tank oficial del Ministerio de Relaciones Exteriores (Instituto de Estudios Internacionales de China), en el que presentaba algunos elementos para reinterpretar el tercer pilar de RtoP estableciendo criterios para que el Consejo de Seguridad evalúe la idoneidad de la intervención militar y garantice su monitoreo y supervisión (Leal Rinaldi y Soreanau Pecequilo, 2021).
} 
la resolución 1970 del Consejo de Seguridad y se abstuvo frente a la resolución 1973 que autorizó la acción militar, China no volvió a avalar la aplicación de la norma de RtoP para otros escenarios, como por ejemplo el de Siria. Por el contrario, a través de la noción de Protección Responsable se fomenta la idea de que una intervención debe ser motivada por razones enteramente humanitarias, que la estabilidad regional debe ser objeto de protección a la par del pueblo, entiende que los medios de protección no militares como los esfuerzos diplomáticos requiere más tiempo pero produce resultados duraderos con menores efectos secundarios y recupera la responsabilidad en la etapa de reconstrucción (Garwood-Gowers, 2016).

El tercer y último curso de acción por el cual China muestra su voluntad de participar en la gobernanza de la agenda global de Seguridad internacional es la jerarquización de hecho que hace de la Organización para la Cooperación de Shanghái (OCS) como instrumento regional. Si bien esta organización originariamente no se postula como una alianza o bloque militar debido a su composición geográfica, de facto se ha erigido en una instancia que contribuye a evitar la intromisión de Estados Unidos y la OTAN en los conflictos y escenarios de disputa regionales (Serbin, 2019). Desde esta perspectiva, Beijing hasta hace poco tiempo se resistía a otorgarle preponderancia a la impronta militar de la OCS para que no sea percibida por Occidente como una amenaza ni se genere una situación que favorezca el incremento de poder relativo de Rusia en su interior, aun así los niveles de interacción y cooperación en esa dimensión han ido increyendo (State Council of Information Office, 2018). De hecho el mismo Xi Jinping reconoció en 2018 que la organización era "una fuerza importante para garantizar la seguridad regional, favorecer el desarrollo conjunto y mejorar la administración global” (Sputniknews, 2018) lo cual, además, está en consonancia con lo indicado en el Libro Blanco Política de China de 2017 sobre la importancia de la cooperación internacional multilateral en la región de Asia y el Pacífico.

Consecuentemente con lo hasta aquí expuesto, está claro que la mayor asertividad de China con respecto a la Seguridad internacional es una tendencia geopolítica de impacto mundial, en sintonía con el proceso de reconfiguración del orden internacional. La misma, además, se despliega de manera acompasada con el descenso no querido de Estados Unidos cuya situación en términos de poderío estratégico-militar dista de ser la de inicios de la posguerra fría. Esto, más allá de que Washington todavía mantiene una ventaja desproporcionada en lo relativo a sus capacidades de poder duro y de influencia con respecto al resto de las potencias mundiales, que lo posicionan en un lugar de superioridad estratégica absoluta, materializada en sus posibilidades de proyectar poder globalmente. 
De este modo, Estados Unidos posee un presupuesto de defensa que es el más alto a nivel mundial y en 2020 representó el 39\% del total invertido en el mundo mientras que China lo sigue con el $13 \%$ por lo que la brecha es muy significativa (Sipri, 2021). Asimismo, el arsenal nuclear es significativamente más grande que el de Beijing y solo comparable al de Moscú, cuenta con 11 portaviones y 10 portahelicópteros dejando muy atrás a Rusia y China (Global Firepower, 2021) y retiene una ventaja tecnológica en áreas clave como la recolección de inteligencia, los misiles balísticos y los aviones de combate de última generación (Marcus, 2019). Finalmente, Estados Unidos cuenta con una red de alianzas en Asia, Europa y América Latina y el Caribe y sus aproximadamente entre 700 y 800 bases militares que se extienden en todo el globo (Vine, 2015).

Pese a esto, desde hace casi dos décadas, la imagen internacional y la legitimidad del liderazgo político de la Casa Blanca, incluso al interior de Occidente, se ha ido erosionando como producto de un comportamiento externo unilateral, militarista y con manifiesto desapego por las reglas del Derecho Internacional Público y los criterios que el mismo Estados Unidos contribuyó a delinear para la gobernanza en los asuntos de paz y seguridad internacional luego de la segunda guerra mundial. El punto más álgido de dicho deterioro y que implicó una aceleración del proceso fue la invasión a Irak en 2003, aunque luego acontecieron otros eventos que siguieron esta lógica como la operación en Libia en 2011 y el abandono del acuerdo nuclear con Irán en 2018 entre los más destacados.

Adicionalmente, la erosión del liderazgo norteamericano en los asuntos de Seguridad internacional se enlazó a la evaluación de los escasos resultados positivos obtenidos por la política de lucha contra el terrorismo internacional, cuya impronta militarista demostró no ser del todo eficaz. Prueba de ello es, por ejemplo, la evolución compleja e incierta que tuvieron escenarios como el de Afganistán e Irak; la multiplicación de atentados durante la primera década del siglo XXI en Europa (Madrid, Londres, Paris); el hecho de no haberse podido evitar ni suprimir el derrame de inestabilidad y violencia terrorista luego del derrocamiento de Gadafi en la región africana de Sahel; incluso, la misma constitución del Daesh en Siria e Irak.

No obstante este contexto poco auspicioso, la vocación de Estados Unidos por liderar el sistema internacional y no ceder su posición de poder en la estructura mundial no puede ser puesta en tela de juicio. La misma quedó plasmada en la Estrategia de Defensa Nacional expedida durante la administración de D. Trump en la que, luego diez años de silencio, Washington dejo asentado que entre sus objetivos de Defensa está continuar siendo la potencia militar predominante en el mundo y asegurar que los balances de poder les sean favorables (Depto. De Defensa de EE. UU., 
2018). Esto en un contexto internacional en el que se percibe que la amenaza principal dejo de ser el terrorismo, como lo sugirió la última Estrategia de Seguridad Nacional al abandonar el énfasis en las amenazas no estatales y mencionar reiteradamente a China (Depto. De Defensa de EE. UU., 2017). De este modo, Estados Unidos sitúa la amenaza en la competencia estratégica interestatal, reconociendo a China y Rusia como potencias revisionistas que buscan moldear el mundo en función de sus modelos autoritarios lo cual ha devenido en un entorno complejo y volátil en función del declive del orden mundial sustentado en normas. En función de esto se avizoran tres cursos de acción direccionados a continuar traccionando para que su poder militar sea el más letal, reforzar las alianzas tradicionales y atraer a más socios y favorecer una reforma al interior del Departamento de Defensa con miras a optimizar su funcionamiento (Torres Sospedra, 2019).

Esta determinación, pertinente con el contexto de descenso no querido que vivencia Estados Unidos, tiende casi de modo ineludible a colisionar con el imparable avance de China que, en los asuntos de Seguridad internacional, deviene evidente en escenarios estratégicos diversos como Asía Central y el Ártico, además de la proyección anteriormente mencionada sobre el Indo Pacífico. Consecuentemente, las relaciones a nivel de estas dos grandes potencias si bien por distintos motivos no son susceptibles, al menos por ahora, de establecer un orden similar al de la guerra fría, presentan como rasgos predominantes la complejidad, la tensión y la competencia, traduciéndose en un clima global de incertidumbre y de potencial escalada en las tensiones.

\section{La vulnerabilidad de América del Sur desde la perspectiva de la Seguridad internacional}

América del Sur, en función de su historia, características y especificidades puede ser comprendida como un complejo de seguridad regional, en términos de B. Buzan y O. Weber (2003), dado que desde una perspectiva analítica-descriptiva la dinámica de seguridad que se despliega en su interior puede ser analizada de manera integral. En este sentido, por ejemplo, se observa cierto patrón de relaciones entre los estados devenido de sus vínculos cooperativos y conflictivos como así también una polaridad múltiple que ha delineado históricamente la disputa por el liderazgo regional que, si bien no se manifiestan abiertamente, es una constante entre los socios mayores (Calderón, 2011). Asimismo, ese patrón ha sido influido desde la inmediata posguerra fría por el desarrollo de las amenazas no tradicionales, entre ellas el crimen 
transnacional organizado, expresado especialmente en el tráfico de drogas y armas y los grupos armados ilegales.

En el marco de esta lógica, se puede observar también que América del Sur está atravesada por tres tendencias que condicionan la recepción de los influjos geopolíticos sistémicos que, a simple vista y en el corto plazo, parecieran estar lejos de impactar por estas latitudes pero que no se pueden minimizar.

La primera tendencia es la complejización de los escenarios de seguridad pública en términos individuales de los estados así como de la subregión en su conjunto. Esto se produce en un contexto de debilidad de los mecanismos de diálogo político y de desencuentro, en el mejor de los casos, de las dirigencias políticas sudamericanas. Al respecto, cabe recordar que durante los últimos años los gobiernos del giro a la derecha desarticularon la Unión de Naciones de América del Sur (UNASUR) en cuyo seno se había sabido activar un comportamiento cooperativo por parte de los distintos poderes ejecutivos en pos de hallar la salida a diferentes situaciones de tensión y hasta conflicto que se sucedieron. Algunas de ellas fueron la acontecida entre Ecuador, Colombia y Venezuela por el bombardeo en Angostura en 2008; entre Colombia y el resto de la subregión por el arrendamiento de bases militares a Estados Unidos en 2009; al interior de Bolivia debido a los intentos de secesión de la región de Cochabamba; y en Ecuador enlazado al motín policial contra el presidente R. Correa. Asimismo, si bien se instauró el PROSUR como instancia para abordar la situación en Venezuela, el mismo no logró articular una dinámica fluida entre los estados, cuyas dirigencias políticas se vieron prontamente abocadas a contener y detener las protestas de sus sociedades ligadas a demandas económicas-sociales, como se pudo observar durante el segundo semestre de 2019 en Ecuador, Perú, Colombia y, en su máxima expresión, en Chile. Luego, el contexto instaurado por el estallido de la pandemia por el virus Covid-19 obligó a los gobiernos a replegarse sobre sí mismos de manera más intensa, al tiempo que se reinstauraron algunas brechas políticasideológicas a partir de las elecciones presidenciales en Argentina y Bolivia y el posterior triunfo de la izquierda para liderar la reforma constitucional en Chile.

En este contexto de carencia de diálogo político subregional promediando el 2021 se articula, por un lado, el incremento de las tensiones y el derrame de violencia en la frontera colombo-venezolana alimentadas por el deterioro acelerado de los acuerdos de paz establecidos en Bogotá durante 2016 y la situación de crisis estructural que atraviesa Caracas desde hace unos años con el gobierno de N. Maduro. En este caso puntual el ambiente extremadamente volátil es producto de la combinación de las disputas territoriales para controlar el tráfico de drogas a ambos lados de la frontera, el flujo de refugiados venezolanos hacia el país vecino que 
asciende a 1.7 millones de personas (ACNUR, 2021), la presencia del Ejército de Liberación Nacional y de los dos frentes de las Fuerzas Armadas Revolucionarias de Colombia que rechazaron los acuerdos de 2016 junto a otros 15 grupos armados aproximadamente que existen en la zona de frontera, sumado a los operativos militares individuales de ambos estados para intentar ejercer su autoridad.

Por otro lado, se suma la continuidad de las situaciones de protesta social en algunas grandes urbes con movilizaciones masivas de las sociedades que, en ocasiones, dan paso a expresiones violentas que abren la puerta a escaladas cuyos desenlaces son muy inciertos como en el caso de Chile en 2019 y Colombia en 2021. $\mathrm{Al}$ respecto, es notorio el incremento de los niveles de represión por parte del Estado que en el 2020, año donde preponderó la cuarentena y el toque de queda como medida paliatoria frente al avance de la pandemia, hubo 210 muertos en las protestas a lo largo de toda la región según Amnistía Internacional, figurando entre los países con mayor cantidad de fallecidos Venezuela, Chile, Bolivia y Ecuador (Lozano, 2021).

Por último, también converge el incremento de índices de violencia criminal que indican la urgencia de la agenda de seguridad pública alimentada por los circuitos ilegales, especialmente el narcotráfico. De este modo, entre muchos ejemplos, se puede mencionar el ingreso de Chile al ranking de los países con puertos de mayor tránsito de droga hacia Europa luego de Colombia, Brasil y Ecuador; el resurgir de Sendero Luminoso en Perú; la formación de grupos armados que disputan el control territorial de la frontera norte de Ecuador; la continuidad del accionar del Ejercito Popular del Pueblo en Paraguay; el asesinato de ambientalistas, líderes sociales y defensores de los derechos humanos especialmente en Colombia y Brasil y la presencia trasnacional del Primer Comando de la Capital brasileño controlando la ruta de tráfico de cocaína que pasa por Perú, Bolivia y Paraguay.

Una segunda tendencia es la continuidad en el tipo de relacionamiento que tiene Estados Unidos con la región latinoamericana en general en lo que respecta a las cuestiones de Seguridad internacional desde la inmediata posguerra fría y que deja traslucir su autopercepción y comportamiento como una potencia hegemónica hemisférica y su vocación por liderar.

El rol de Washington desde una perspectiva estratégico-militar tiene una intensidad y permanencia que se ancla en el despliegue territorial de sus capacidades estratégicas, de inteligencia y financiamiento y de un esquema de influencias muy significativo en lo referido a las relaciones con las instituciones castrenses. Asimismo, la Casa Blanca es la que propone iniciativas y planes de acción a los gobiernos, manteniendo y fortaleciendo los canales de asistencia financiera directa y de venta de armas y los programas de entrenamiento y educación para las Fuerzas Armadas y de 
seguridad (Calderón, 2015). Este despliegue, por consiguiente, ilustra su vocación de liderar los asuntos de Seguridad internacional sostenida en el tiempo pero, siempre, constante e inobjetable.

Así, por ejemplo, en los noventa Washington propuso la noción de seguridad cooperativa como modelo para las relaciones interamericanas, instauró la Conferencia de ministros de Defensa de las Américas y fortaleció la presencia del Comando Sur. Luego, en la primera década del siglo XXI esa voluntad de liderazgo coexistió, particularmente en América del Sur, con el discurso autonómico de los gobiernos del giro a la izquierda, y se expresó en el reclamo pos 11S a los estados para que ejerzan una mayor vigilancia de sus fronteras y territorios, y en el compromiso estratégico con Colombia para abordar el conflicto armado interno. Finalmente, en la segunda década del siglo XXI, durante la primera parte Estados Unidos reafirma su vocación por liderar con decisiones como la de convocar la Primera Reunión de Organizaciones Militares Interamericanas, sus propuestas para la Junta Interamericana de Defensa y el accionar con relación al gobierno de Venezuela percibido como una amenaza a la estabilidad regional a partir del decreto ejecutivo de 2015. Durante el segundo quinquenio con el giro a la derecha de América del Sur y la posterior llegada de D. Trump a la presidencia, Washington se encontró con un contexto subregional más receptivo a sus políticas. El mismo le permitió respaldar la creación del Grupo de Lima y el PROSUR, contribuyendo indirectamente al ocaso del regionalismo posneoliberal (Legler, 2013); también ejecutar la Operación América Unida instalando un base militar temporal en Tabatinga; acceder a la base de Alcántara en Brasil; nombrar un general brasilero como vicecomandante del Comando Sur responsable de la asistencia humanitaria y sumar al país carioca como aliado extra-OTAN.

Finalmente, la tercera tendencia versa en la desatención de la Defensa nacional como un área estratégica en la mayoría de los estados sudamericanos debido, en buena medida, a la reconfiguración del rol de sus Fuerzas Armadas a partir de la inmediata posguerra fría en torno al abordaje de la agenda de seguridad pública signada por el narcotráfico como amenaza más relevante.

Esa reconfiguración tuvo lugar en un contexto subregional con un bajo nivel de conflictividad interestatal, el avance de los mecanismos de diálogo político, la escasez de recursos para invertir en Defensa y las cada vez más acuciantes demandas económicas y sociales. De igual modo, cabe considerar también la circunstancia de que el retorno de la democracia se desarrolló de manera heterogénea en los estados sudamericanos, con instituciones militares que mayoritariamente preservaron cuotas de poder e influencia importantes sobre el sistema político, limitando los procesos de 
construcción de la supremacía civil e imposibilitando el despliegue de la conducción política de la Defensa como un ámbito estratégico del Estado. De hecho, solo por mencionar algunos ejemplos significativos, se puede constatar que los sistemas de justicia penal militar continúan vigentes excepto en Argentina y Ecuador que derogaron la jurisdicción militar; en el ámbito económico sobreviven situaciones de privilegio como el caso de las Fuerzas Armadas ecuatorianas que poseen decenas de empresas en rubros estratégicos desvinculados de la Defensa o la continuidad de la Ley del Cobre en Chile hasta 2018; en cuanto a la permanencia de la capacidad de influencia sobre el proceso de toma de decisiones, el caso de Brasil cuyos militares se reacomodaron a las nuevas instancias de asesoramiento y asistencia. De este modo, buena parte del proceso de transformación llevado adelante en las instituciones militares y con relación a su vínculo con el Poder Ejecutivo y los ministerios de Defensa, fue más de forma que de fondo y convivieron con una preparación muy deficiente, en el mejor de los casos, de cuadros civiles especializados (Calderón, 2020).

Para concluir, es relevante mencionar también que, desde hace algunos años, a ese escenario de desatención de la Defensa se le adiciona los pedidos de colaboración de las dirigencias políticas a los militares para gestionar situaciones de alta conflictividad que hacen peligrar la estabilidad de sus mandatos. Estos, por lo general, se debilitan rápidamente debido a la complejidad de los contextos nacionales enlazados con problemas económicos-sociales crónicos, institucionalidades débiles y la creciente movilización de la sociedad civil. En función de ello y como dato contextual hay que considerar que desde 2008 los gobiernos de la región latinoamericana, en general, cuentan con escasos niveles de aceptación a poco de iniciar sus mandatos y las denominadas lunas de miel con sus respectivas sociedades suelen durar cada vez menos (Latinobarómetro, 2018). Este denominado nuevo militarismo (Diamint 2015; Verde Montenegro Escanéz, 2019) o militarismo cívico (Rodríguez, 2018) se erige en un proceso que eclosionó en 2019 en el contexto de las protestas sociales de varios de los estados de Sudamérica y, definitivamente, aleja a las Fuerzas Armadas de su misión principal y a los estados de conducir la Política de Defensa como una política pública estratégica.

Consecuentemente, dado los procesos intrasubregionales en curso se puede aseverar que la situación de América del Sur es de extrema vulnerabilidad frente a una posible escalada de tensión en el vínculo entre Estados Unidos y China en el ámbito de la Seguridad internacional porque sus posibilidades de mantenerse al margen de la puja de poder de ambas potencias son muy mínimas. Por ello, el desafío analítico más acuciante es sopesar en qué medida los procesos en curso en Sudamérica 
pueden profundizar tales vulnerabilidades frente a la proyección de poder que hagan ambas potencias.

\section{Sudamérica: lejos pero no tanto, secundaria pero no irrelevante}

Como se adelantó en la introducción de este escrito, cuando se reflexiona acerca de las tendencias geopolíticas globales en el contexto de la reconfiguración del sistema internacional y del orden en términos estratégico-militares, sólo en circuitos académicos muy específicos, como la Universidad de la Defensa Nacional en Argentina, se analiza el impacto sobre la región latinoamericana o, específicamente, en América del Sur. Esto puede atribuirse a la lectura estratégica que se efectúa de la región como irrelevante o bien a la vigencia de su condición de patio trasero de Estados Unidos.

No obstante, en el primer caso y desde una perspectiva geopolítica, es decir considerando el impacto de la geografía sobre la política y las Relaciones Internacionales, la trascendencia y el peso de la región es inobjetable en función de, por ejemplo, la biodiversidad, los aproximadamente 30 acuíferos existentes, los recursos minerales, de gas y petróleo y la proyección a la Antártida entre otros múltiples elementos (CEED, 2015).Por otra parte, en lo relativo al vínculo con Washington, si bien es cierto que la región es percibida como su zona de influencia natural y en consecuencia el despliegue que se realiza tiene una magnitud que lo torna rotundo, también lo es el hecho de que la presencia de China en distintos nichos estratégicos como el comercio internacional, los grandes proyectos de infraestructura, el campo de la tecnología y el ámbito de la financiación internacional es cada vez más relevante. De hecho, de la totalidad de estados sudamericanos, 6 tienen acuerdos de asociación estratégica y asociación estratégica integral con Beijing (Bejano, Malenay Velloso, 2015) y 8 han adherido a la Iniciativa OBOR. Además, para 2017 China se convirtió en el primer socio comercial de todos los países de la subregión en función del volumen de sus exportaciones y es el segundo socio comercial de América Latina después de Estados Unidos. Finalmente, entre 2007 y 2017 las inversiones de Beijing en la región aumentaron en 25000 millones de dólares superando a las realizadas por Estados Unidos y la Unión Europea, con el plus de que los bancos chinos operan con estados que poseen una baja calificación crediticia (Grabendorf, 2018). Además, los 5 principales beneficiarios de préstamos chinos entre 2007 y 2016 de América Latina son sudamericanos (Vásquez, 2019) y Argentina, Ecuador y Uruguay han ingresado entre 2019 y 2020 al Banco Asiático de 
Inversión en Infraestructura que tiene a Brasil como miembro fundador desde 2020 y aún esperan como candidatos Perú, Bolivia, Chile y Venezuela.

Por lo tanto, entendiendo que Sudamérica es un espacio estratégico por diferentes motivos ligados a sus recursos naturales y posicionamiento geográfico y a sabiendas de que crecimiento de la presencia de China es parte de su estrategia diplomática y geopolítica global y, por ende, es intensa y dinámica (Girado, 2017), puede visualizarse que los estados de la subregión cuentan con muy pocas chances de poder desarrollar un comportamiento internacional que los mantenga al margen de la puja de poder entre Washington y Beijing. Este estado de cosas se replica también en lo relativo al ámbito de la Seguridad internacional y si se considera el condicionamiento que ejercen los procesos mencionados en el apartado anterior que atraviesan a América del Sur, la situación de los países es de extrema vulnerabilidad. Por lo tanto, es factible esbozar al menos dos interrogantes.

El primero de ellos se enlaza a dos de los procesos antes mencionados que se despliegan en Sudamérica relativos a la volatilidad de la seguridad individual de los estados y de la subregión como conjunto y a la vocación de liderazgo de Estados Unidos con relación a las cuestiones de Seguridad internacional. En este sentido, considerando que los intereses de China son contundentes y se hallan en permanente expansión, resulta relevante preguntarse con respecto al escenario que se puede configurar en el caso de que tales intereses sean afectados por un deterioro mayor de la seguridad en los países o en la subregión, o bien en función del comportamiento que Washington puede promover en tales estados o, incluso, adoptar en su calidad de potencia regional.

Con relación a este interrogante, resulta pertinente observar el comportamiento que ha desplegado Beijing durante los últimos años en otras latitudes, específicamente Asía Central y, en menor medida, África. En estos escenarios, si bien son completamente diferentes y es imposible extrapolarlos a América del Sur, China, ha dado muestras de su interés por, como mínimo, no obviar las cuestiones de Seguridad internacional en tanto ámbito de relacionamiento con los gobiernos de aquellos estados donde tiene una presencia económica y financiera.

En Asía Central, por ejemplo, se pueden encontrar varios indicios que ilustran cómo en un lapso breve Beijing ha entablado relaciones en el ámbito de la seguridad con todos los estados de la región, incluso pese al rol eminentemente estratégico que desempeña Rusia y la circunstancia de que es su zona de influencia histórica y, en un punto, la más neurálgica. De este modo, desde mediados de los años 90 China ha sabido construir un vínculo cooperativo con Moscú en torno a esta región, materializado en la OCS el cual, desde 2001 especialmente, se reforzó con el objetivo 
de contrarrestar la presencia de Estados Unidos a partir de la apertura del escenario bélico en Afganistán. Así se estableció una suerte de división del trabajo por la cual mientras Moscú opera como proveedor de seguridad, Beijing avanza comercial y económicamente en el contexto de su iniciativa OBOR, dado que Asia Central es percibida como una tierra de oportunidades en cuanto a sus recursos naturales y las posibilidades de desarrollar proyectos e infraestructura (Lemon, 2018). De este modo, China se convirtió en el mayor socio económico de muchos de esos estados, liderando las inversiones y respaldando las deudas de sus gobiernos.

Sin embargo, aproximadamente desde mediados de la segunda década del siglo XXI, el gobierno de Xi Jinpingha ido construyendo lazos de seguridad con todas las repúblicas de Asia Central a través de diferentes instrumentos como los acuerdos sobre defensa, la realización de ejercicios militares, el ofrecimiento de ayuda militar no compensada, la presencia de compañías de seguridad privadas en territorios vecinos y la venta de sistemas de defensa aéreos, drones para uso militar y tecnologías y hardware militares de origen chino ${ }^{5}$. Incluso, se confirmaron los rumores sobre la presencia china en una base cerca de la frontera con Tayikistan lo cual valida su intención de expandir su rol como proveedor de seguridad en la región, más allá de la incomodidad que pueda generar en su vínculo con otras potencias, en este caso, India (Shih,2019) $)^{6}$. Por consiguiente, se puede corroborar el avance incipiente pero concreto de China en la dimensión estratégico-militar de las Relaciones Internacionales, incluso en un escenario en el cual, además de colisionar con los intereses norteamericanos, le puede suponer abrir un frente de tensiones con Rusia dado que constituye su esfera de influencia más próxima y en la que ha desarrollado un liderazgo histórico.

En lo que se refiere al continente africano, en los últimos años la penetración china a través de lo que se conoce como modelo de Angola ${ }^{7}$ parece estar incorporando

\footnotetext{
${ }^{5}$ A modo de ejemplos se puede relevar: los ejercicios militares organizados en la OCS con Tajikistan en 2016 y 2019, que es el primer receptor de ayuda militar no compensada para reforzar los puestos de frontera con Afganistán y abrir un centro de contraterrorismo y también recibe a en su territorio a las compañías de seguridad privada chinas; los 10 ejercicios militares realizados con Kirgistan entre 2003 y 2016 y en 2019 ejecutaron el Cooperation 2019 en una base de Xinjiang contra el terrorismo; un acuerdo militar con Kazajstán en 2015 para realizar ejercicios de contraterrorismo e insurgencia; Turkmenistan y Uzbekistan compraron sistemas de defensa aéreos y drones para uso militar; todos los estados han adquirido tecnologías y hardware militares de origen chino (Lemon, 2018)

${ }^{6}$ En el caso puntual de la presencia china en Tayikistan, esto incomoda a Nueva Delhi porque esa base era el único punto de acceso en función del conflicto que mantienen con Pakistán por Cachemira.

${ }^{7}$ El modelo Angola consistente en el acercamiento de Beijing a los estados poseedores de hidrocarburos y minerales para financiar obras públicas e infraestructuras a un costo inferior al de los mercados internacionales, incluso recibiendo pagos en recursos.
} 
muy lentamente la dimensión de la seguridad en función del reconocimiento de la necesidad de defender sus intereses económicos y a la ciudadanía residente en el continente. Esto puede hallar explicación en el Libro Blanco sobre Estrategia Militar de China donde se asevera que “...la seguridad de los intereses de ultramar relacionados con la energía y recursos, líneas de comunicaciones marítimas estratégicas, así como de instituciones, personal y activos en el exterior, se ha convertido en un problema inminente." (Gobierno de China, 2015).

Al respecto, entonces, se puede observar que en 2017 Beijing abrió la primera base militar en África, en Djibouti, donde se ubican también otros contingentes militares extranjeros de Estados Unidos y Francia, incluso existen rumores sobre la apertura de nuevas bases en los próximos años (Mateos, 2019). Asimismo en 2018 auspició el primer Foro China-África sobre Defensa y Seguridad, en el cual 50 países establecieron sus prioridades para los próximos años frente al gobierno chino. En este sentido, una de las amenazas que china comparte con los estados de la costa occidental del continente negro es la piratería, en este caso, contra activos y ciudadanos chinos frente a lo cual Beijing procura profundizar la cooperación en seguridad marítima específicamente con los países de la región del Golfo de Guinea a través de distintos mecanismos como reuniones de alto nivel hasta el entrenamiento antipiratería para las Fuerzas Armadas (Gonzalez Levaggi, 2020). De hecho, la Armada China busca participar en operaciones contra piratería usando su base en Djibuti, siendo relevante el Ejercicio Mosi realizado con Rusia y Sudáfrica con el objetivo de “...garantizar la seguridad del transporte marítimo y la actividad económica marítima." (Panda, 2018). Igualmente importante es el avance de Beijing como proveedor de sistemas de armas dado que entre 2006 y 2010 se convirtió en el mayor exportador hacia el África subsahariana, superando a Rusia y siendo Nigeria su principal cliente, mientras que entre 2014 y 2018 fue responsable del 24\%de tales exportaciones (Mateos, 2019). Finalmente, Beijing ha demostrado su voluntad por jugar un rol significativo con relación a las OMP y, específicamente, apoyando a la UA.

Queda claro entonces que en ambas regiones, aunque con intensidades muy diferentes, China ha comenzado a incursionar en el ámbito de la Seguridad internacional como espacio de relacionamiento con los gobiernos de aquellos estados donde tiene una presencia económica y financiera. Por ende, no se puede descartar que en el mediano plazo, quizás bajo otro formato y con instrumentos y mecanismos similares, pueda configurarse un escenario parecido en América del Sur en virtud de la intensidad y dinamismo de la presencia china y de la circunstancia de que la situación de seguridad en la subregión se orienta hacia una mayor complejizacion. 
De hecho la Seguridad internacional como ámbito de interés en el vínculo de Beijing con Sudamérica queda registrada oficialmente por primera vez en el documento Política China hacia América Latina y el Caribe de 2016, el cual contiene un apartado denominado Área de la paz, la seguridad y la justicia, con un punto específico sobre intercambio y cooperación militar que estaba ausente en el primero de su tipo expedido en 2008 (Valenzuela Alvaréz, 2017). No obstante, durante los años precedentes tuvieron lugar una serie de eventos indicativos de este interés como en 2015 la realización bajo patrocinio chino del Foro sobre Logística Militar del cual participaron 11 estados latinoamericanos, la instalación de la estación de observación espacial en Argentina y los acuerdos en el área de Defensa concretados entre Beijing y 8 de los 10 estados sudamericano entre 2010 y 2016 (Resdal, 2016).

Por otra parte, un segundo interrogante a considerar se vincula a la colisión de los intereses norteamericanos y chinos en el Atlántico Sur, en torno a la navegación y la accesibilidad de las rutas de comercio, los recursos ictícolas y energéticos y el acceso a la Antártida. Al mismo tiempo, se enlaza con el tercer proceso subregional explicitado en este artículo, vinculado a la desatención relativa de la Defensa nacional como un área estratégica en la mayoría de los estados sudamericanos. En este caso, la pregunta sería acerca de las posibilidades y herramientas político-diplomáticas y estratégico-militares que pueden llegar a desplegar los estados sudamericanos para mantenerse al margen en una situación de escalada en la puja de poder entre las potencias en torno al Atlántico Sur.

Un ejemplo contundente del arribo de esta lógica de disputa y su impacto en la subregión se ha dado en los primeros meses del 2021 con el lanzamiento por parte de Estados Unidos de la Operación Cruz del Sur con Guyana, Brasil, Uruguay y Argentina, a realizarse con el buque Stone de la Guardia Costera norteamericana. El objetivo anunciado era garantizar que el hemisferio occidental sea seguro, libre y próspero, para lo cual se postulaba como necesario, entre otras cuestiones, contrarrestar la pesca ilegal, no regulada y no declarada y esto implicaba que el buque Stone realizase tareas de patrullaje en las zonas económica exclusiva de los países participantes (Infobae, 2021).

El asunto es que el Operativo Cruz del Sur tiene lugar en un momento en que las declaraciones de jefe del Comando Sur Craig Faller señalan a China como la mayor amenaza junto con los grupos delictivos internacionales (Natalizio, 2021). Asimismo, los documentos de los últimos 5 años de distintos entes del gobierno de Estados Unidos advierten sobre la presencia de Beijing en el Atlántico Sur y su capacidad de proyectar poder así como acerca de sus prácticas económicas predatorias y el lugar que ocupa mundialmente como el estado que más desarrolla la pesca ilegal (Tokatlian, 
2021). De hecho en 2016, el Consejo Nacional de Inteligencia del país del norte publicó un informe donde definió la pesca ilegal como una amenaza para la seguridad alimentaria y económica que beneficia al crimen organizado transnacional y, en 2020,la nueva estrategia del Servicio de la Guardia Costera indicó que dicha práctica había sustituido a la piratería como principal amenaza a la seguridad marítima y que "...socavan las democracias libres y abiertas, desafían la seguridad y la prosperidad y desestabilizan los países en riesgo en todo el mundo" (US Embassy Chile, 2020).

Hay que considerar, también, que China viene asomándose al océano Atlántico Sur desde hace ya varios años en función de su relevancia geopolítica ligada, como se advirtió más arriba, a los recursos naturales oceánicos, las rutas de comercio y la proyección a la Antártida. En primer término Beijing, como importador neto del sector energético con cuotas en permanente ascenso de las cuales el 52\% son oriundas de Medio Oriente, necesitaba diversificar sus proveedores. Por tanto, el descubrimiento en el siglo XXI de nuevas reservas frente a las costas de África y América Latina significaron que la estrategia china de Going Out llegase a ambas regiones, consolidando y expandiendo las operaciones de sus empresas energéticas que se habían iniciado de forma exploratoria a mediados de los 90 (Vásquez, 2019). De este modo para 2019 África aportaba el 18\% de las importaciones chinas y América Latina el 15\% (Brasil 8\%, Colombia 3\% y Venezuela 2\%) teniendo estas últimas un incremento del 47\% entre 2017 y 2019 (Enernews, 2021). Por otra parte, es sabido que el Atlántico Sur es la mayor reserva pesquera del mundo para desarrollar en gran escala la pesca de captura en un contexto en el cual el resto de los mares se encuentran sobreexplotados y que Beijing como principal demandante de la producción ictícola tiene la flota oceánica de pesqueros de mayor envergadura (Castro, 2010). En este escenario los reclamos contralas prácticas ilegales de las embarcaciones de bandera china son cuantiosos por parte de los estados costeros y de organizaciones no gubernamentales como Greenpeace, así también se multiplican los incidentes como en 2016 cuando Prefectura Naval Argentina hundió un buque chino dentro de su zona económica exclusiva y en 2020 detuvo otros 3 junto con la Armada y en 2017 cuando 8 buques fueron retenidos por inspectores de Guinea, Sierra Leona y GuineaBissau.

En segundo lugar, el gigante asiático busca a garantizar un uso seguro de las rutas marítimas de comunicación en función de la expansión de su comercio internacional y las inversiones externas directas en África y América Latina. Al respecto es significativo el trabajo realizado por la empresa China Communications Construction Company en el cuarto puente sobre el Canal de Panamá, el Puerto de Veracruz en Mendoza, el Puerto Sur en Brasil y el puerto de Santiago en Cuba; 
asimismo esta empresa tiene intenciones tanto de participar en el dragado de la hidrovía del Río Paraná como en la construcción de un puerto de aguas profundas en Uruguay (Gonzalez Levaggi, 2020). Asimismo, es relevante conocer que las empresas chinas tienen presencia en 46 puertos africanos a través de su financiamiento, construcción o su manejo operativo, estando 28 en la costa atlántica, de los cuales 6 son operados por empresas chinas.

Finalmente, la presencia militar china en el Atlántico Sur se concreta en las costas africanas con la participación china en las OMP y las acciones de la diplomacia militar en todo el continente que representan el 11\% del total de las llevadas adelante por Beijing, siendo los principales socios de la costa occidental Sudáfrica, Namibia, Angola y Nigeria (Gonzalez Levaggi, 2020). Esto además de los puntos mencionados anteriormente con respecto a la relevancia de los asuntos de seguridad en el modo de vinculación que despliega china con el continente negro. Por otra parte, en lo relativo a las costas del Atlántico frente a la región latinoamericana la cooperación militar crece, aunque con mayor lentitud y América del Sur representa el 8\% del total de las acciones de la diplomacia militar china. Sobresalen también, las reuniones de alto nivel realizadas con Brasil y Argentina entre 2003 y 2016, 29 y 21 respectivamente; los 3 ejercicios militares combinados realizados con Brasil que además recibió 3 visitas de la Armada China; y a fines de 2013 la visita de 3 buques de la Flota del Mar del Sur de la AEPL por primera vez a Chile, Argentina y Brasil atravesando el Estrecho de Magallanes (Gonzalez Levaggi, 2020). Asimismo, se destacan las giras del buque hospital Arca de la Paz que se despliegan desde 2008. Específicamente a América Latina llegó en 2011 (Cuba, Costa Rica, Jamaica y Trinidad y Tobago), en 2015 (México, Barbados, Granada y Perú) y en 2018 (Venezuela) para realizar operaciones de asistencia humanitaria y de socorro en situaciones de desastre, obviamente en consonancia con la intención de Beijing de proyectar una imagen al mundo de potencia responsable (BBC, 2018). Por último, mencionar que desde 2005 China acciona con el objetivo a incrementar su liderazgo con respecto a la cuestión antártica instalando su tercera y cuarta bases de investigación en 2009 y 2014, planeando la construcción de una quinta en el Mar de Ross, reparando el rompe hielo Dragón de Hielo, creando el Instituto de Estudios Antárticos en Shanghái y siendo sede en 2017 de la Reunión Consultiva del Tratado Antártico, entre otras múltiples iniciativas y acciones que dan cuenta de la proyección dura y blanda de Beijing en el continente blanco (Witker, 2018).

En este contexto, entonces, plagado de percepciones adversas, intereses estratégicos que colisionan y búsquedas cruzadas para proyectar poder, queda cada vez más claro que una problemática preocupante como lo es la pesca ilegal en las 
zonas económicas exclusivas de los estados sudamericanos se puede proyectar como el eje de la cooperación y el conflicto en el mediano plazo con las potencias en disputa. Si bien no se puede obviar que esta problemática es real, de larga data y que afecta a los recursos económicos de los estados y la seguridad alimentaria global, tampoco se puede hacer caso omiso al hecho de que involucra a varios estados europeos y algunos otros de Asia además de a Beijing. No obstante, lo central a estas alturas es la circunstancia de que los gobiernos de la subregión quedan expuestos a una disyuntiva de difícil resolución debido a sus escasas posibilidades de llevar adelante un patrullaje eficaz y por sus propios medios. De esta forma, los estados sudamericanos navegan entre declinar o aceptar la iniciativa norteamericana para vigilar los espacios marítimos a sabiendas tanto de las dificultades inherentes a un rechazo como del peligro implícito que ello significa de cara a la posibilidad de que acontezca, accidentalmente o no, alguna situación o roce con pesqueros de bandera china.

Por ello, en el caso de los países de América del Sur con frente marítimo amplios, la situación de desatención de la Defensa como un área estratégica del Estado y la consiguiente debilidad, específicamente, de sus armadas y de los sistemas que le permiten desplegar el control y vigilancia de sus espacios marítimos, impacta negativamente en las posibilidades de los gobiernos de mantenerse al margen de la puja de poder entre las potencias y sus intentos de proyectar poder.

\section{A modo de conclusión}

A lo largo de este escrito se pudo observar cómo las tendencias geopolíticas mundiales cobran nitidez en torno a la mayor asertividad de China en el ámbito de la Seguridad internacional expresada en sus capacidades estratégico-militares y en su voluntad de participar en la gobernanza de la agenda global relativa a esos asuntos, en un contexto de retroceso relativo y no deseado de Estados Unidos. La complejidad inherente a la coexistencia de Beijing y Washington como los estados de mayor relevancia del sistema internacional desde una perspectiva integral está fuera de toda discusión como así también el impacto que esto conlleva para el resto de los países, entre ellos los sudamericanos, en cuanto a la dimensión estratégico-militar. De ahí que sea factible avizorar tiempos inciertos.

Asimismo, en lo concerniente a América del Sur, partiendo desde un acercamiento teórico-conceptual interméstico, se advierte que la situación de los estados es de extrema vulnerabilidad frente a una posible escalada de tensión en el vínculo entre las potencias en el ámbito de la Seguridad internacional. Esto acontece en función de las escasas posibilidades que tienen los gobiernos de mantenerse al 
margen de la puja de poder debido, en buena medida, al contexto configurado a partir de los procesos intrasubregionales en curso.

Dado este estado de cosas, entonces, es inevitable preguntarse sobre los escenarios posibles frente a una afectación relevante de los intereses de China en Sudamérica, sea por un deterioro mayor de la seguridad en los países o en la subregión, o bien debido al comportamiento que Washington puede promover en tales estados o adoptar en su calidad de potencia regional. Asimismo, en torno al espacio del Atlántico Sur como escenario estratégico, emerge la inquietud acerca de las posibilidades y herramientas político-diplomáticas y estratégico-militares que pueden llegar a desplegar los estados sudamericanos para mantenerse al margen en una situación de escalada en la puja de poder entre Estados Unidos Y China.

En breves, de cara al mediano plazo la labor investigativa académica debiese orientarse a intentar dilucidar chasta qué punto la asertividad de China en el ámbito de la Seguridad internacional puede profundizar la situación de vulnerabilidad de América del Sur? La respuesta, si bien tentativa, pareciera no ser alentadora si se observa el modo en que Beijing se vincula y comporta en otras latitudes. Por consiguiente, ahondar los esfuerzos en ese sentido deviene imperioso.

\section{Referencias}

Acharya, A. (1999). The periphery as the core: the third world and security studies. Centre for International and Strategic Studies, occasional paper number 28, York University.

ACNUR (2021), Situación en Venezuela. Recuperado de: https://www.acnur.org/situacion-en-venezuela.html

Ayoob, M. (1995). Subaltern realism: International relations meets the Third World. International relations and the Third World (pp. 31-54). St. Martin Press.

BBC (2018). Cómo es el "Arca de la Paz", el buque hospital que China envió a Venezuela para prestar asistencia sanitaria por una semana. Recuperado de: https://www.bbc.com/mundo/noticias-america-latina-45634232

Baqués Quesada, J. (2019). El mar como catalizador de la geopolítica: de Mahan al auge chino.Revista de Estudios en Seguridad internacional, volumen 5, No. 1 (pp. 119-139). Recuperado de: http://www.seguridadinternacional.es/resi/index.php/revista/article/view/1 17

Berjano Ramón, C.; Malena J. y Velloso Miguel A. (2015). El relacionamiento de China con América Latina y argentina significado de la alianza estratégica 
integral y los recientes acuerdos bilaterales.Documentos de Trabajo 96 CARI. Recuperado de: https://www.cari.org.ar/pdf/dt96.pdf

Buzan, B. y Waever, O. (2003). Regions and Powers: The Security of International Security. Cambridge UniversityPress.

Calderón, E. (2020). Ocaso del regionalismo posneoliberal en Sudamérica: las deudas de la marea rosa en la conducción política de la Defensa, Íconos, número 68 (pp. 19-37).

- (2015). Estados Unidos y el combate contra el narcoterrorismo como lineamiento de política exterior central en el vínculo con América Latina: el caso del modelo de cooperación con Colombia, Cuadernos de Política Exterior Argentina, número 122(pp.1-42).

- (2011). Brasil en el Consejo de Defensa Sudamericano: obstáculos a la cooperación y el liderazgo. Relaciones Internacionales, número 18 (pp89-111).

Castro, J. (2010). EL Atlántico Sur en el contexto mundial, simposio El Atlántico Sur, visiones y posturas, Boletín del Centro Naval, número 827. Recuperado de: https://www.centronaval.org.ar/boletin/BCN827/827-CASTRO.pdf

CEED (Centro de Estudios Estratégicos de Defensa) (2015). Estudio Prospectivo Sudamérica 2025, Consejo de Defensa Sudamericano. Recuperado de: https://es.scribd.com/document/390728034/UNASUR-Estudio-ProspectivoSuramerica-2025

Depto. De Defensa de EEUU. Estrategia de Defensa Nacional (2018). Gobierno de Estados Unidos. Recuperado de: https://www.defense.gov/Explore/Spotlight/National-Defense-Strategy/

Departamento de Defensa de EEUU. Estrategia de Seguridad Nacional (2017). Gobierno de Estados Unidos. Recuperado de: https://www.defense.gov/Explore/Spotlight/National-Defense-Strategy/

Departamento de Seguridad Nacional (2019). El Libro Blanco de la República Popular de China sobre la Defensa Nacional en la nueva era. Recuperado de: https://www.dsn.gob.es/es/actualidad/sala-prensa/libro-blancorep\%C3\%BAblica-popular-china-sobre-defensa-nacional-nueva-era-2019

Diamint. R. (2015). A new militarism in Latin America. Journal of Democracy, $\mathrm{n}^{\circ} 26$ (pp. 155-168). Recuperado de: https://journalofdemocracy.org/articles/anew-militarism-in-latin-america/

Enernews, (2021). Poder de China: Franja y Ruta de la Seda las cuentas energéticas de China con América Latina. Recuperado de: http://enernews.com/poder-de- 
china/337944/franja-y-ruta-de-la-seda-las-cuentas-energeticas-de-china-conamerica-latina

Escudé, C. (1995). El realismo de los Estados débiles. Grupo Editor Latinoamericano.

Estatuto del Consejo de Defensa Suramericano de la UNASUR (2008). Recuperado de: http://unasursg.org/es/documentos-normativos-unasur.html

Garwood-Gowers, A. (2016). China's 'responsible protection' concept: Reinterpreting the Responsibility to Protect (R2P) and military intervention for humanitarian purposes. Asian Journal of International Law, número 6 (pp. 89118). Recuperado de: https://www.cambridge.org/core/journals/asianjournal-of-international-law/article/abs/chinas-responsible-protectionconcept-reinterpreting-the-responsibility-to-protect-r2p-and-militaryintervention-for-humanitarianpurposes/6E3EC0292AAA64C21F0C1C7ECAB2F415

Girado, G. (noviembre, 2017). América Latina en la estrategia económica china. Entrevista realizada por Mariano Schuster para NUSO.org. Recuperado de: https://nuso.org/articulo/america-latina-en-la-estrategia-economica-china/

Global Firepower (2021). Recuperado de: https://www.globalfirepower.com/

GonzalezLevaggi, A. (2020), Eurasia en el Atlántico Sur: evaluando la proyección marítima de China, Rusia e India, Revista Defensa Nacional, volumen 5. Recuperado de: https://www.undef.edu.ar/libros/wpcontent/uploads/2021/04/GONZA\%CC\%81LEZ-LEVAGGI.-DefensaNacional-Nro.-5-arti\%CC\%81culo-3-1.pdf

Grabendorf, W. (2018). América Latina en la era Trump ¿Una región en disputa entre Estados Unidos y China? NUSO número 275. Recuperado de: https://nuso.org/articulo/america-latina-en-la-era-trump/

Hevia Fernández, B. (2020). China: Operaciones de Mantenimiento de la Paz en África. IEEE, documento de opinión 53. Recuperado de: http://www.ieee.es/Galerias/fichero/docs_opinion/2020/DIEEEO53_2020B EAHEV_misionesChina.pdf y/o enlace bie3 
Holsti, K. (1995). International relations theory and domestic war in the Third World: The limits of relevance. International relations and the Third World (pp. 103-132). St. Martin Press.

Gobierno de China. Libro Blanco sobre Estrategia Militar de China 2015. Recuperado http://english.www.gov.cn/archive/white_paper/2015/05/27/content_28147 5115610833.htm

Infobae (2021), Estados Unidos brindó nuevos detalles de la "Operación Cruz del Sur" para combatir la pesca ilegal en el Atlántico Sur. Recuperado de: https://www.infobae.com/america/eeuu/2021/01/15/estados-unidosbrindo-nuevos-detalles-de-la-operacion-cruz-del-sur-para-combatir-la-pescailegal-en-el-atlantico-sur/

Latinobarómetro (2018). Informe 2018. Recueprado de: https://www.latinobarometro.org/lat.jsp

Leal Rinaldi, A. y SoreanauPecequilo, C. (2021). The Contemporary World Order, BRICS, and the R2P Principle: the Cases of Brazil and China (2005/2017). Colombia Internacional 105 (pp. 3-28). Recuperado de: http://www.scielo.org.co/pdf/rci/n105/0121-5612-rci-105-3.pdf

Legler, T. (2013). Post-Hegemonic Regionalism and Sovereignty in Latin America: Optimists, Skeptics, and an Emerging Research Agenda. ContextoInternacional, numero 2 (pp.181-208).

Lemon, E. J. (2018). China, Russia and Security in Central Asia, Rising Powers in Global Governance. Disponible en https://risingpowersproject.com/chinarussia-security-central-asia/

Lozano, D. (2021). América Latina, la región más violeta y represiva del mundo. El Mundo. Recuperado de: https://www.elmundo.es/internacional/2020/02/27/5e56c39c21 efa09f408b4 71b.html

Marcus, J. (2019). Cómo el poderío de China hace peligrar el dominio militar de EE.UU. en el Pacífico, BBC. Recuperado de: https://www.bbc.com/mundo/noticias-internacional-49473519 
Mateos, O. (2019). Carrera Internacional por la Seguridad en África, Anuario Internacional Anual CIDOB (pp.172-179). Recuperado de: https://raco.cat/index.php/AnuarioCIDOB/article/view/359739

Natalizio, J. (2021). EE.UU.-China y la disputa del Atlántico Sur. Radio Grafica. Recuperado de: https://radiografica.org.ar/2021/04/11/ee-uu-china-y-ladisputa-del-atlantico-sur/

Neuman, S. (1995). International relations theory and the Third World: An oxymoron?.International relations and the Third World (pp. 1-29). St. Martin Press.

Panda, J. P. (2018). India's Call on China in the Quad: A Strategic Arch between Liberal and Alternative Structures. Powers in global governance, volumen 3, Issue 2 (pp. 83-111). Recuperado de: https://risingpowersproject.com/quarterly/indias-call-on-china-in-the-quada-strategic-arch-between-liberal-and-alternative-structures/

Resdal (Red de Estudios de Seguridad y Defensa de América Latina) (2016). Atlas comparativo de Defensa en América Latina y el Caribe 2016. Recuperado de: https://www.resdal.org/index.html

Rodríguez, S.M. (2018). Building civilian militarism: Colombia, internal war, and militarization in a mid-term perspective. Security Dialogue, no 49 (pp. 109-122). https://journals.sagepub.com/doi/10.1177/0967010617743201

Rose, G. (1998). Neoclassical Realism and Theories of Foreign Policy. World Politics, volumen 51, (pp.144-172).

SCIO (State Council of Information Office) (2018). A quick guide to SCO and its military cooperation. Recuperado de: https://english.scio.gov.cn

Serbin, A. (2019). El conflicto entre India y Pakistán. Perfil. Recuperado de: https://www.perfil.com/noticias/columnistas/el-conflicto-entre-india-ypakistan.phtml

Shih, G. (2019). In Central Asia's forbidding highlands, a quiet newcomer: Chinese tropos, The Washington Post. Recuperado de: https://www.washingtonpost.com/world/asia_pacific/in-central-asias- 
forbidding-highlands-a-quiet-newcomer-chinese-

troops/2019/02/18/78d4a8d0-1e62-11e9-a759-2b8541bbbe20_story.html

SIPRI (Stockholm International Peace Research Institute) (2021). Yearbook 2021 Armaments, Disarmament and International Security. Recuperado de: https://www.sipri.org/yearbook/2021

Sputniknews (2018). China constata el papel importante de la OCS para la seguridad regional. Recuperado de: https://mundo.sputniknews.com/20180609/papelde-ocs-en-asia-1079447673.html

Tokatlian, J. G. (2021). El Comando Sur, China y las Malvinas. El cohete a la luna. Recuperado de: https://www.elcohetealaluna.com/el-comando-sur-china-ylas-malvinas/

Torres Sospedra, J. (2019). La Estrategia de Defensa Nacional estadounidense: el retorno de la historia y el fin de la utopía. IEEE, documento de opinión 01. Recuperado de: https://dialnet.unirioja.es/servlet/articulo?codigo=6962177

Valenzuela Alvaréz, J. L. (2017). Evolución de la Política Exterior de China para América Latina. Relaciones Internacionalesn 53 (pp.115- 133). Recuperado de: https://revistas.unlp.edu.ar/RRII-IRI/article/view/3626

Vásquez, P. (2019). La huella hidrocarburífera de China en América Latina y África. Open Edition Journals. Recuperado de: https://journals.openedition.org/poldev/3272

Verdes-Montenegro Escánez, F. J. (2019). La (re)militarización de la política latinoamericana. Origen y consecuencias para las democracias de la región. Documentos de Trabajo Fundación Carolina ${ }^{\circ} 14$ (2a época). Recuperado de: https://www.fundacioncarolina.es/wpcontent/uploads/2019/09/DT_FC_14.pdf

Vine D. (2015). Base Nation: How U.S. Military Bases Abroad Harm America and the World. MetropolitanBooks.

UN (UnitedNations), (2021a). Mantenimiento de la Paz. Recuperado de: https://peacekeeping.un.org/es/how-we-are-funded 
UN (UnitedNations), (2021b), Mantenimiento de la Paz. Recuperado de: https://peacekeeping.un.org/es/troop-and-police-contributors

US Emabasy Chile (2020). Nueva estrategia para combatir la pesca ilegal. Recuperado de: https://cl.usembassy.gov/es/ee-uu-inicia-una-nueva-estrategia-paracombatir-la-pesca-ilegal/

Witker, I. (2018).El Programa Antárticode la República Popular China:proyecciones duras y blandas.Revista de REDCAEM, número 7. Recuperado de: https://www.cepal.org/sites/default/files/events/files/sesion_3._ivan_witker. pdf 